Article

\title{
Existence of Solutions for Some Coupled Systems of Fractional Differential Inclusions
}

\author{
Aurelian Cernea ${ }^{\dagger}$
}

Faculty of Mathematics and Computer Science, University of Bucharest, 010014 București, Romania; acernea@fmi.unibuc.ro

+ Shared authorship: Academy of Romanian Scientists, Splaiul Independenţei 54, 050094 Bucharest, Romania.

Received: 12 April 2020; Accepted: 27 April 2020; Published: 2 May 2020

check for updates

\begin{abstract}
We study two coupled systems of nonconvex fractional differential inclusions with certain nonlocal boundary conditions and we prove the existence of solutions in the case when the set-valued maps are Lipschitz in the state variables.
\end{abstract}

Keywords: fractional derivative; differential inclusion; boundary condition

MSC: 34A60; 34A08; 34B15

\section{Introduction}

The last decades represent a period of intense development of the qualitative theory of fractional differential equations and of fractional differential inclusions [1-5] etc. The justification is that these mathematical objects seem to be very useful in the research of several physical phenomena. Recently, several papers were devoted to the study of coupled systems of fractional differential equations. Such kinds of coupled systems have an important applicability in many and different natural situations.

In the literature, we find several definitions for fractional integrals and for fractional derivatives. From the large number of definitions that exist for fractional derivatives the most used in applied problems are the standard Riemann-Liouville fractional derivative and Caputo fractional derivative. Concrete problems need definitions of fractional derivative allowing to use initial conditions that have physically meanings. This requirement is satisfied by Caputo fractional derivative, introduced in [6].

The present paper is motivated by the fact that in recent papers [7,8] it was obtained, via fixed point techniques, the existence of solutions for some coupled systems of fractional differential equations with some nonlocal non-separated boundary conditions.

Our goal is to extend the studies in [7,8] to the set-valued framework. More precisely, we consider first the problem

$$
\begin{aligned}
& \begin{cases}D_{C}^{\alpha} u(t) \in F(t, u(t), v(t)) & \text { a.e. }[0,1], \\
D_{C}^{\beta} v(t) \in G(t, u(t), v(t)) & \text { a.e. }[0,1],\end{cases} \\
& \left\{\begin{array} { l } 
{ u ( 0 ) = \lambda _ { 1 } v ( 1 ) , } \\
{ v ( 0 ) = \mu _ { 1 } u ( 1 ) , }
\end{array} \quad \left\{\begin{array}{l}
D_{C}^{\gamma} u(1)=\lambda_{2} D_{C}^{\gamma} v(\xi), \\
D_{C}^{\gamma} v(1)=\mu_{2} D_{C}^{\gamma} u(\xi),
\end{array}\right.\right.
\end{aligned}
$$

where $\alpha, \beta \in(1,2], \gamma, \xi \in(0,1), \mu_{i}, \lambda_{i} \in \mathbf{R}, i=\overline{1,2}, \mu_{1} \lambda_{1} \neq 1, \mu_{2} \lambda_{2} \xi^{2(1-\gamma)} \neq 1, D_{c}^{q}$ is the Caputo fractional derivative of order $q, F:[0,1] \times \mathbf{R} \times \mathbf{R} \rightarrow \mathcal{P}(\mathbf{R})$ and $G:[0,1] \times \mathbf{R} \times \mathbf{R} \rightarrow \mathcal{P}(\mathbf{R})$ are given set-valued maps.

Next, we deal with the problem

$$
\begin{cases}D^{\sigma} u(t) \in F(t, u(t), v(t)) & \text { a.e. }[0,1] \\ D^{\phi} v(t) \in G(t, u(t), v(t)) & \text { a.e. }[0,1]\end{cases}
$$




$$
\begin{aligned}
& \left\{\begin{array}{l}
u(0)=u^{\prime}(0)=\ldots=u^{(n-2)}(0)=0, \\
\int_{0}^{1} u(s) d s=\sum_{i=2}^{p_{1}} a_{i-1} \int_{\eta_{i-1}}^{\eta_{i}} v(s) d s+\sum_{j=1}^{q_{1}} b_{j} v\left(\rho_{j}\right),
\end{array}\right. \\
& \left\{\begin{array}{l}
v(0)=v^{\prime}(0)=\ldots=v^{(n-2)}(0)=0, \\
\int_{0}^{1} v(s) d s=\sum_{i=2}^{p_{2}} c_{i-1} \int_{\theta_{i-1}}^{\theta_{i}} u(s) d s+\sum_{j=1}^{q_{2}} d_{j} u\left(\zeta_{j}\right),
\end{array}\right.
\end{aligned}
$$

where $n-1<\sigma<n, m-1<\phi<m, n \geq 3, m \geq 3, D^{r}$ is the standard Riemann-Liouville fractional derivative of order $r, a_{i}, b_{i}, c_{i}, d_{i}>0,0<\eta_{1}<\ldots<\eta_{p_{1}}<\ldots<\rho_{1}<\ldots<\rho_{q_{1}}<1$, $0<\theta_{1}<\ldots<\theta_{p_{2}}<\ldots<\zeta_{1}<\ldots<\zeta_{q_{2}}<1, p_{1}, q_{1}, p_{2}, q_{2} \in \mathbf{N}, F:[0,1] \times \mathbf{R} \times \mathbf{R} \rightarrow \mathcal{P}(\mathbf{R})$ and $G:[0,1] \times \mathbf{R} \times \mathbf{R} \rightarrow \mathcal{P}(\mathbf{R})$ are given set-valued maps.

We study the situation when the values of $F$ and $G$ are not convex. Instead of convexity, our assumption is that $F$ and $G$ are Lipschitz in the second and third variable. We adapt the ideas in [9] in order to obtain the existence of solutions for problems (1) and (2) and (3)-(5). In the set-valued framework when it is studied a differential inclusion without convexity in the right-hand side, Filippov's theorem [9] provides the existence of solutions starting from a given mapping which is called "quasi" solution or "almost" solution. Moreover, the result contains an estimate between the "quasi" solution and the solution.

It is worth to mention that from a such kind of result for a particular choice of the known almost solution we obtain an existence result which look less complicated. These consequences may also be obtained using Covitz-Nadler set-valued contraction principle [10] but we avoided to present them since there are less powerfull than Filippov's type approach: weaker hypotheses and no a priori bounds for solutions. Moreover, in this particular case, if the set-valued maps are reduced to single-valued maps then we cover similar resuts existing in the literature, namely in $[7,8]$.

Such kind of results may be found in the literature ([11-14] etc.), but their presentation in the framework of coupled systems of differential inclusions is new.

\section{Preliminaries}

Consider $(Y, d)$ a metric space. The Pompeiu-Hausdorff distance of the closed subsets $M, N \subset Y$ is defined by

$$
d_{H}(M, N)=\max \left\{d^{*}(M, N), d^{*}(N, M)\right\}, d^{*}(M, N)=\sup \{d(a, N) ; a \in M\},
$$

with $d(x, N)=\inf _{y \in N} d(x, y)$.

In what follows $I=[0,1]$ and $C(I, \mathbf{R})$ is the Banach space of all continuous functions from $I$ to $\mathbf{R}$. Its norm is denoted by $\|x(.)\|_{C}=\sup _{t \in I}|x(t)| . L^{1}(I, \mathbf{R})$ denotes the space of functions $u():. I \rightarrow \mathbf{R}$ that are integrable. This Banach space is endowed with the norm $\|u(.)\|_{1}=\int_{0}^{1}|u(t)| d t$. In this paper, with $\chi_{S}(\cdot)$ we denote the characteristic function of the set $S \subset \mathbf{R}$.

We recall the next definitions that are well known in the fractional calculus.

The fractional integral of order $p>0$ of a mapping $f:(0, \infty) \rightarrow \mathbf{R}$ is

$$
I^{p} f(t)=\int_{0}^{t} \frac{(t-s)^{p-1}}{\Gamma(p)} f(s) d s .
$$

In the above definition $f$ is Lebesgue integrable and $\Gamma$ is the (Euler's) Gamma function; namely, $\Gamma(p)=\int_{0}^{\infty} t^{p-1} e^{-t} d t$.

The Riemann-Liouville fractional derivative of order $p>0$ of a continuous map $f:(0, \infty) \rightarrow \mathbf{R}$ is given by

$$
D^{p} f(t)=\frac{1}{\Gamma(n-p)} \frac{d^{n}}{d t^{n}} \int_{0}^{t}(t-s)^{-p+n-1} f(s) d s,
$$

where $n=[p]+1$. 
The Caputo fractional derivative of order $p>0$ of $f:[0, \infty) \rightarrow \mathbf{R}$ is defined by

$$
\mathrm{D}_{c}^{p} f(t)=\frac{1}{\Gamma(n-p)} \int_{0}^{t}(t-s)^{-p+n-1} f^{(n)}(s) d s,
$$

where $n=[p]+1$.

In the last two definitions we implicitly assume that $f$ is $n$ times differentiable and its $n$-th derivative is absolutely continuous.

Lemma 1. For given $f(),. g(.) \in L^{1}(I, \mathbf{R})$, the unique solution $(u, v)$ of problem

$$
\left\{\begin{array}{l}
D_{C}^{\alpha} u(t)=f(t) \\
D_{C}^{\beta} v(t)=g(t)
\end{array}\right.
$$

with boundary conditions (2) is given by

$$
\begin{aligned}
& u(t)=\frac{\mu_{2} \Gamma(2-\gamma)}{\Delta}\left[\frac{\lambda_{1}\left(\mu_{1} \lambda_{2} \xi^{1-\gamma}+1\right)}{1-\lambda_{1} \mu_{1}}+\lambda_{2} \xi^{1-\gamma} t\right] A_{3}-\frac{\Gamma(2-\gamma)}{\Delta}\left[\frac{\lambda_{1}\left(\mu_{1} \lambda_{2} \xi^{1-\gamma}+1\right)}{1-\lambda_{1} \mu_{1}}+\lambda_{2} \xi^{1-\gamma} t\right] B_{3}+ \\
& \frac{\lambda_{2} \Gamma(2-\gamma)}{\Delta}\left[\frac{\lambda_{1}\left(\mu_{1}+\mu_{2} \xi^{1-\gamma}\right)}{1-\lambda_{1} \mu_{1}}+t\right] B_{2}-\frac{\Gamma(2-\gamma)}{\Delta}\left[\frac{\lambda_{1}\left(\mu_{1}+\mu_{2} \xi^{1-\gamma}\right)}{1-\lambda_{1} \mu_{1}}+t\right] A_{2}+\frac{\lambda_{1}}{1-\lambda_{1} \mu_{1}}\left(\mu_{1} A_{1}+B_{1}\right)+\int_{0}^{t} \frac{(t-s)^{\alpha-1}}{\Gamma(\alpha)} f(s) d s \\
& v(t)=\frac{\mu_{2} \Gamma(2-\gamma)}{\Delta}\left[\frac{\mu_{1}\left(\lambda_{1}+\lambda_{2} \xi^{1-\gamma}\right)}{1-\lambda_{1} \mu_{1}}+t\right] A_{3}-\frac{\Gamma(2-\gamma)}{\Delta}\left[\frac{\mu_{1}\left(\lambda_{1}+\lambda_{2} \xi^{1-\gamma}\right)}{1-\lambda_{1} \mu_{1}}+t\right] B_{3}+\frac{\lambda_{2} \Gamma(2-\gamma)}{\Delta}\left[\frac{\mu_{1}\left(\lambda_{1} \mu_{2} \xi^{1-\gamma}+1\right)}{1-\lambda_{1} \mu_{1}}+\right. \\
& \left.\mu_{2} \xi^{1-\gamma} t\right] B_{2}-\frac{\Gamma(2-\gamma)}{\Delta}\left[\frac{\mu_{1}\left(\lambda_{1} \mu_{2} \xi^{1-\gamma}+1\right)}{1-\lambda_{1} \mu_{1}}+\mu_{2} \xi^{1-\gamma} t\right] A_{2}+\frac{\mu_{1}}{1-\lambda_{1} \mu_{1}}\left(A_{1}+\lambda_{1} B_{1}\right)+\int_{0}^{t} \frac{(t-s)^{\beta-1}}{\Gamma(\beta)} g(s) d s,
\end{aligned}
$$

where $\Delta=1-\mu_{2} \lambda_{2} \xi^{2(1-\gamma)}, A_{1}=\int_{0}^{1} \frac{(1-s)^{\alpha-1}}{\Gamma(\alpha)} f(s) d s, B_{1}=\int_{0}^{1} \frac{(1-s)^{\beta-1}}{\Gamma(\beta)} g(s) d s, A_{2}=\int_{0}^{1} \frac{(1-s)^{\alpha-\gamma-1}}{\Gamma(\alpha-\gamma)} f(s) d s$, $B_{2}=\int_{0}^{\xi} \frac{(\xi-s)^{\beta-\gamma-1}}{\Gamma(\beta-\gamma)} g(s) d s, A_{3}=\int_{0}^{\xi} \frac{(\xi-s)^{\alpha-\gamma-1}}{\Gamma(\alpha-\gamma)} f(s) d s$ and $B_{3}=\int_{0}^{1} \frac{(1-s)^{\beta-\gamma-1}}{\Gamma(\beta-\gamma)} g(s) d s$.

Proof. The proof may be found in [8], namely Lemma 2.1.

Remark 1. If we denote $c_{1}(t)=\frac{\Gamma(2-\gamma)}{\Delta}\left[\frac{\lambda_{1}\left(\mu_{1} \lambda_{2} \xi^{1-\gamma}+1\right)}{1-\lambda_{1} \mu_{1}}+\lambda_{2} \xi^{1-\gamma} t\right], c_{2}(t)=\frac{\Gamma(2-\gamma)}{\Delta}\left[\frac{\lambda_{1}\left(\mu_{1}+\mu_{2} \xi^{1-\gamma}\right)}{1-\lambda_{1} \mu_{1}}+t\right]$, $c_{3}(t)=\frac{\Gamma(2-\gamma)}{\Delta}\left[\frac{\mu_{1}\left(\lambda_{1}+\lambda_{2} \xi^{1-\gamma}\right)}{1-\lambda_{1} \mu_{1}}+t\right], c_{4}(t)=\frac{\Gamma(2-\gamma)}{\Delta}\left[\frac{\mu_{1}\left(\lambda_{1} \mu_{2} \xi^{1-\gamma}+1\right)}{1-\lambda_{1} \mu_{1}}+\mu_{2} \xi^{1-\gamma} t\right]$,

$$
\begin{gathered}
H_{1}(t, s)=\frac{\lambda_{1} \mu_{1}}{1-\lambda_{1} \mu_{1}} \frac{(1-s)^{\alpha-1}}{\Gamma(\alpha)}-c_{2}(t) \frac{(1-s)^{\alpha-\gamma-1}}{\Gamma(\alpha-\gamma)}+\frac{(t-s)^{\alpha-1}}{\Gamma(\alpha)} \chi_{[0, t]}(s)+\mu_{2} c_{1}(t) \frac{(\xi-s)^{\alpha-\gamma-1}}{\Gamma(\alpha-\gamma)} \chi_{[0, \xi]}(s) \\
H_{2}(t, s)=\frac{\lambda_{1}}{1-\lambda_{1} \mu_{1}} \frac{(1-s)^{\beta-1}}{\Gamma(\beta)}-c_{1}(t) \frac{(1-s)^{\beta-\gamma-1}}{\Gamma(\beta-\gamma)}+\lambda_{2} c_{2}(t) \frac{(\xi-s)^{\beta-\gamma-1}}{\Gamma(\beta-\gamma)} \chi_{[0, \xi]}(s), \\
H_{3}(t, s)=\frac{\mu_{1}}{1-\lambda_{1} \mu_{1}} \frac{(1-s)^{\alpha-1}}{\Gamma(\alpha)}-c_{4}(t) \frac{(1-s)^{\alpha-\gamma-1}}{\Gamma(\alpha-\gamma)}+\mu_{2} c_{3}(t) \frac{(\xi-s)^{\alpha-\gamma-1}}{\Gamma(\alpha-\gamma)} \chi_{[0, \xi]}(s), \\
H_{4}(t, s)=\frac{\lambda_{1} \mu_{1}}{1-\lambda_{1} \mu_{1}} \frac{(1-s)^{\beta-1}}{\Gamma(\beta)}-c_{3}(t) \frac{(1-s)^{\beta-\gamma-1}}{\Gamma(\beta-\gamma)}+\frac{(t-s)^{\beta-1}}{\Gamma(\beta)} \chi_{[0, t]}(s)+\lambda_{2} c_{4}(t) \frac{(\xi-s)^{\beta-\gamma-1}}{\Gamma(\beta-\gamma)} \chi_{[0, \xi]}(s)
\end{gathered}
$$

then the solution $(u(),. v()$.$) in Lemma 1$ may be written as

$$
\begin{array}{ll}
u(t)=\int_{0}^{1} H_{1}(t, s) f(s) d s+\int_{0}^{1} H_{2}(t, s) g(s) d s, & t \in I \\
v(t)=\int_{0}^{1} H_{3}(t, s) f(s) d s+\int_{0}^{1} H_{4}(t, s) g(s) d s, \quad t \in I .
\end{array}
$$

Moreover, if $\alpha>\gamma+1$ and $\beta>\gamma+1$, for any $t, s \in$ I one has

$$
\left|H_{1}(t, s)\right| \leq \frac{1}{\Gamma(\alpha)}\left(1+\left|\frac{\lambda_{1} \mu_{1}}{1-\lambda_{1} \mu_{1}}\right|\right)+\frac{1}{\Gamma(\alpha-\gamma)}\left(\max _{t \in[0,1]}\left|c_{2}(t)\right|+\left|\mu_{2}\right| \xi^{\alpha-\gamma-1} \max _{t \in[0,1]}\left|c_{1}(t)\right|\right)=: M_{1},
$$




$$
\begin{gathered}
\left|H_{2}(t, s)\right| \leq \frac{1}{\Gamma(\beta)}\left|\frac{\lambda_{1}}{1-\lambda_{1} \mu_{1}}\right|+\frac{1}{\Gamma(\beta-\gamma)}\left(\max _{t \in[0,1]}\left|c_{1}(t)\right|+\left|\lambda_{2}\right| \xi^{\beta-\gamma-1} \max _{t \in[0,1]}\left|c_{2}(t)\right|\right)=: M_{2}, \\
\left|H_{3}(t, s)\right| \leq \frac{1}{\Gamma(\alpha)}\left|\frac{\mu_{1}}{1-\lambda_{1} \mu_{1}}\right|+\frac{1}{\Gamma(\alpha-\gamma)}\left(\max _{t \in[0,1]}\left|c_{4}(t)\right|+\left|\mu_{2}\right| \xi^{\alpha-\gamma-1} \max _{t \in[0,1]}\left|c_{3}(t)\right|\right)=: M_{3}, \\
\left|H_{4}(t, s)\right| \leq \frac{1}{\Gamma(\beta)}\left(1+\left|\frac{\lambda_{1} \mu_{1}}{1-\lambda_{1} \mu_{1}}\right|\right)+\frac{1}{\Gamma(\beta-\gamma)}\left(\max _{t \in[0,1]}\left|c_{3}(t)\right|+\left|\lambda_{2}\right| \xi^{\beta-\gamma-1} \max _{t \in[0,1]}\left|c_{4}(t)\right|\right)=: M_{4} .
\end{gathered}
$$

Definition 1. The functions $u(.) \in C(I, \mathbf{R})$ and $v(.) \in C(I, \mathbf{R})$ are called a solution of problem (1) and (2) if there exist functions $f(),. g(.) \in L^{1}(I, \mathbf{R})$ that satisfies $f(t) \in F(t, u(t), v(t))$ a.e. (I), $g(t) \in G(t, u(t), v(t))$ a.e. (I) and $(u(),. v()$.$) is given by (6).$

In the definition above it is implicitly assumed that the Caputo derivative of order $\alpha$ of $u($.$) exists$ on $[0,1]$ and the Caputo derivative of order $\beta$ of $v($.$) exists on [0,1]$.

Lemma 2. Consider $f(),. g(.) \in L^{1}(I, \mathbf{R})$, define

$$
\Lambda_{1}=\frac{1}{\phi} \sum_{i=2}^{p_{1}} a_{i-1}\left(\eta_{i}^{\phi}-\eta_{i-1}^{\phi-1}\right)+\sum_{j=1}^{q_{1}} b_{j} \rho_{j}^{\phi-1}, \quad \Lambda_{2}=\frac{1}{\sigma} \sum_{i=2}^{p_{2}} c_{i-1}\left(\theta_{i}^{\sigma}-\theta_{i-1}^{\sigma-1}\right)+\sum_{j=1}^{q_{2}} d_{j} \zeta_{j}^{\phi-1},
$$

and assume that $\Omega=\frac{1}{\sigma \phi}-\Lambda_{1} \Lambda_{2} \neq 0$.

Then the solution of the system

$$
\left\{\begin{array}{l}
D_{C}^{\alpha} u(t)=f(t) \\
D_{C}^{\beta} v(t)=g(t)
\end{array}\right.
$$

with boundary conditions (4) and (5) is given by

$$
\begin{aligned}
& u(t)=\frac{1}{\Gamma(\sigma)} \int_{0}^{t}(t-s)^{\sigma-1} f(s) d s+\frac{t^{\sigma-1}}{\Omega}\left\{\frac { 1 } { \phi } \left(\sum_{i=2}^{p_{1}} a_{i-1} \frac{1}{\Gamma(\phi)} \int_{\eta_{i-1}}^{\eta_{i}} \int_{0}^{s}(s-\tau)^{\phi-1} g(\tau) d \tau d s\right.\right. \\
& \left.+\sum_{j=1}^{q_{1}} b_{j} \frac{1}{\Gamma(\phi)} \int_{0}^{\rho_{j}}\left(\rho_{j}-s\right)^{\phi-1} g(s) d s-\frac{1}{\Gamma(\sigma)} \int_{0}^{1} \int_{0}^{t}(t-s)^{\sigma-1} f(s) d s d t\right)+\Lambda_{1}\left(\sum_{i=2}^{p_{2}} c_{i-1} \frac{1}{\Gamma(\sigma)} \int_{\theta_{i-1}}^{\theta_{i}}\right. \\
& \left.\left.\int_{0}^{s}(s-\tau)^{\phi-1} f(\tau) d \tau d s+\sum_{j=1}^{q_{2}} d_{j} \frac{1}{\Gamma(\sigma)} \int_{0}^{\zeta_{j}}\left(\zeta_{j}-s\right)^{\sigma-1} f(s) d s-\frac{1}{\Gamma(\phi)} \int_{0}^{1} \int_{0}^{t}(t-s)^{\phi-1} g(s) d s d t\right)\right\}, \\
& v(t)=\frac{1}{\Gamma(\phi)} \int_{0}^{t}(t-s)^{\phi-1} g(s) d s+\frac{t^{\phi-1}}{\Omega}\left\{\frac { 1 } { \sigma } \left(\sum_{i=2}^{p_{2}} c_{i-1} \frac{1}{\Gamma(\sigma)} \int_{\theta_{i-1}}^{\theta_{i}} \int_{0}^{s}(s-\tau)^{\phi-1} f(\tau) d \tau d s\right.\right. \\
& \left.+\sum_{j=1}^{q_{2}} d_{j} \frac{1}{\Gamma(\sigma)} \int_{0}^{\zeta_{j}}\left(\zeta_{j}-s\right)^{\sigma-1} f(s) d s-\frac{1}{\Gamma(\phi)} \int_{0}^{1} \int_{0}^{t}(t-s)^{\phi-1} g(s) d s d t\right)+\Lambda_{2}\left(\sum_{i=2}^{p_{1}} a_{i-1} \frac{1}{\Gamma(\phi)} \int_{\eta_{i-1}}^{\eta_{i}}\right. \\
& \left.\left.\int_{0}^{s}(s-\tau)^{\phi-1} g(\tau) d \tau d s+\sum_{j=1}^{q_{1}} d_{j} \frac{1}{\Gamma(\phi)} \int_{0}^{\rho_{j}}\left(\rho_{j}-s\right)^{\phi-1} g(s) d s-\frac{1}{\Gamma(\sigma)} \int_{0}^{1} \int_{0}^{t}(t-s)^{\sigma-1} f(s) d s d t\right)\right\} .
\end{aligned}
$$

Proof. The proof may be found in [7], namely Lemma 2.1.

Remark 2. If we denote

$$
\begin{aligned}
& K_{1}(t, s)=\frac{1}{\Gamma(\sigma)}(t-s)^{\sigma-1} \chi_{[0, t]}(s)-\frac{t^{\sigma-1}}{\Omega \Gamma(\sigma) \sigma \phi}\left[(1-s)^{\sigma}-s^{\sigma}\right]+\frac{\Lambda_{1}}{\Gamma(\sigma) \sigma} \sum_{i=2}^{p_{2}} c_{i-1}\left[\left(\theta_{i}-s\right)^{\sigma}-\right. \\
& \left.\left(\theta_{i-1}-s\right)^{\sigma}\right] \chi_{\left[0, \theta_{i}\right]}(s)+\frac{\Lambda_{1}}{\Gamma(\sigma)} \sum_{j=1}^{q_{2}} d_{j}\left(\zeta_{j}-s\right)^{\sigma-1} \chi_{\left[0, \zeta_{j}\right]}(s), \\
& K_{2}(t, s)=\frac{t^{\sigma-1}}{\Omega \Gamma(\phi) \phi^{2}} \sum_{i=2}^{p_{1}} a_{i-1}\left[\left(\eta_{i}-s\right)^{\phi}-\left(\eta_{i-1}-s\right)^{\phi}\right] \chi_{\left[0, \eta_{i}\right]}(s)+\frac{t^{\sigma-1}}{\Omega \Gamma(\phi) \phi} \sum_{j=1}^{q_{1}} b_{j}\left(\rho_{j}-s\right)^{\phi-1} \chi_{\left[0, \rho_{j}\right]}(s) \\
& -\frac{\Lambda_{1} t^{\sigma-1}}{\Omega \Gamma(\phi) \phi}\left[(1-s)^{\phi}-s^{\phi}\right], \\
& K_{3}(t, s)=\frac{t^{\phi-1}}{\Omega \Gamma(\sigma) \sigma^{2}} \sum_{i=2}^{p_{2}} c_{i-1}\left[\left(\theta_{i}-s\right)^{\sigma}-\left(\theta_{i-1}-s\right)^{\sigma}\right] \chi_{\left[0, \theta_{i}\right]}(s)+\frac{t^{\phi-1}}{\Omega \Gamma(\sigma) \sigma} \sum_{j=1}^{q_{2}} d_{j}\left(\zeta_{j}-s\right)^{\sigma-1} \chi_{\left[0, \zeta_{j}\right]}(s) \\
& -\frac{\Lambda_{2} t^{\phi-1}}{\Omega \Gamma(\sigma) \sigma}\left[(1-s)^{\sigma}-s^{\sigma}\right], \\
& K_{4}(t, s)=\frac{1}{\Gamma(\phi)}(t-s)^{\phi-1} \chi_{[0, t]}(s)-\frac{t^{\phi-1}}{\Omega \Gamma(\phi) \sigma \phi}\left[(1-s)^{\phi}-s^{\phi}\right]+\frac{\Lambda_{2}}{\Gamma(\phi) \phi} \sum_{i=2}^{p_{1}} a_{i-1}\left[\left(\eta_{i}-s\right)^{\phi}-\right. \\
& \left.\quad\left(\eta_{i-1}-s\right)^{\phi}\right] \chi_{\left[0, \eta_{i}\right]}(s)+\frac{\Lambda_{2}}{\Gamma(\phi)} \sum_{j=1}^{q_{1}} b_{j}\left(\rho_{j}-s\right)^{\phi-1} \chi_{\left[0, \rho_{j}\right]}(s),
\end{aligned}
$$


then the solution $(u(),. v()$.$) in Lemma 2$ may be written as

$$
\begin{array}{ll}
u(t)=\int_{0}^{1} K_{1}(t, s) f(s) d s+\int_{0}^{1} K_{2}(t, s) g(s) d s, & t \in I \\
v(t)=\int_{0}^{1} K_{3}(t, s) f(s) d s+\int_{0}^{1} K_{4}(t, s) g(s) d s, & t \in I .
\end{array}
$$

Moreover, since $\sigma-1 \geq 1, \phi-1 \geq 1$, for any $t, s \in I$, we have

$$
\begin{aligned}
&\left|K_{1}(t, s)\right| \leq \frac{1}{\Gamma(\sigma)}+\frac{2}{|\Omega| \Gamma(\sigma) \sigma \phi}+\frac{\Lambda_{1}}{\Gamma(\sigma) \sigma} \sum_{i=2}^{p_{2}} c_{i-1}\left(\theta_{i}^{\sigma}+\theta_{i-1}^{\sigma}\right)+\frac{\Lambda_{1}}{\Gamma(\sigma)} \sum_{j=1}^{q_{2}} d_{j} \zeta_{j}^{\sigma-1}=m_{1}, \\
&\left|K_{2}(t, s)\right| \leq \frac{1}{|\Omega| \Gamma(\phi) \phi^{2}} \sum_{i=2}^{p_{1}} a_{i-1}\left(\eta_{i}^{\phi}+\eta_{i-1}^{\phi}\right)+\frac{1}{|\Omega| \Gamma(\phi) \phi} \sum_{j=1}^{q_{1}} b_{j} \rho_{j}^{\phi-1}+\frac{2 \Lambda_{1}}{|\Omega| \Gamma(\phi) \phi}=: m_{2}, \\
&\left|K_{3}(t, s)\right| \leq \frac{1}{|\Omega| \Gamma(\sigma) \sigma^{2}} \sum_{i=2}^{p_{2}} c_{i-1}\left(\theta_{i}^{\sigma}+\theta_{i-1}^{\sigma}\right)+\frac{1}{|\Omega| \Gamma(\sigma) \sigma} \sum_{j=1}^{q_{2}} d_{j} \zeta_{j}^{\sigma-1}+\frac{2 \Lambda_{2}}{|\Omega| \Gamma(\sigma) \sigma}=: m_{3}, \\
&\left|K_{4}(t, s)\right| \leq \frac{1}{\Gamma(\phi)}+\frac{2}{|\Omega| \Gamma(\phi) \sigma \phi}+\frac{\Lambda_{2}}{\Gamma(\phi) \phi} \sum_{i=2}^{p_{1}} a_{i-1}\left(\eta_{i}^{\phi}+\eta_{i-1}^{\phi}\right)+\frac{\Lambda_{2}}{\Gamma(\phi)} \sum_{j=1}^{q_{1}} b_{j} \rho_{j}^{\phi-1}=: m_{2} .
\end{aligned}
$$

Definition 2. The pair $(u(),. v().) \in C(I, \mathbf{R}) \times C(I, \mathbf{R})$ is said to be a solution of problem (3)-(5) if there exist functions $f(),. g(.) \in L^{1}(I, \mathbf{R})$ that satisfies $f(t) \in F(t, u(t), v(t))$ a.e. $(I), g(t) \in G(t, u(t)$, v $(t))$ a.e. (I) and $(u(),. v()$.$) is given by (7)$.

Finally, we recall a well known selection result in the set-valued analysis [15].

Lemma 3. Let $Y$ be a separable Banach space, $B$ its closed unit ball, $H: I \rightarrow \mathcal{P}(Y)$ is a multifunction whose values are nonempty closed and $w: I \rightarrow X, s: I \rightarrow \mathbf{R}_{+}$are two measurable mappings. If

$$
H(t) \cap(w(t)+s(t) B) \neq \varnothing \quad \text { a.e. }(I),
$$

then the multifunction $t \rightarrow H(t) \cap(w(t)+s(t) B)$ admits a measurable selection.

\section{The Main Results}

Our results are valid if we make the following assumptions.

Hypothesis 1. (i) $F(., .):. I \times \mathbf{R} \times \mathbf{R} \rightarrow \mathcal{P}(\mathbf{R}), G(. .,):. I \times \mathbf{R} \times \mathbf{R} \rightarrow \mathcal{P}(\mathbf{R})$ have nonempty closed values and are $\mathcal{L}(I) \otimes \mathcal{B}(\mathbf{R} \times \mathbf{R})$ measurable.

(ii) There exist $l(),. m(.) \in L^{1}(I,(0, \infty))$ such that, for almost all $t \in I, F(t, . .$.$) is l(t)$-Lipschitz and $G(t, . .$.$) is m(t)$-Lipschitz in the sense that

$$
\begin{gathered}
d_{H}\left(F\left(t, x_{1}, y_{1}\right), F\left(t, x_{2}, y_{2}\right)\right) \leq l(t)\left(\left|x_{1}-x_{2}\right|+\left|y_{1}-y_{2}\right|\right) \quad \forall x_{1}, x_{2}, y_{1}, y_{2} \in \mathbf{R} . \\
d_{H}\left(G\left(t, x_{1}, y_{1}\right), G\left(t, x_{2}, y_{2}\right)\right) \leq m(t)\left(\left|x_{1}-x_{2}\right|+\left|y_{1}-y_{2}\right|\right) \quad \forall x_{1}, x_{2}, y_{1}, y_{2} \in \mathbf{R} .
\end{gathered}
$$

We use next the following notation: $L(t)=M_{1} l(t)+M_{2} m(t)+M_{3} l(t)+M_{4} m(t), t \in I$. Also, in the next theorem we implicitly assume that the Caputo derivative of order $\alpha$ of $x($.$) exists on [0,1]$ and the Caputo derivative of order $\beta$ of $y($.$) exists on [0,1]$.

Theorem 1. Assume that Hypothesis 1 is satisfied, $\alpha>\gamma+1, \beta>\gamma+1$ and $\|L(.)\|_{1}<1$.

Consider $x(.) \in C(I, \mathbf{R}), y(.) \in C(I, \mathbf{R})$ with $x(0)=\lambda_{1} x(1), y(0)=\mu_{1} x(1) D_{C}^{\gamma} x(1)=\lambda_{2} D_{C}^{\gamma} y(\xi)$, $D_{C}^{\gamma} y(1)=\mu_{2} D_{C}^{\gamma} x(\xi)$, and there exists $p(),. q(.) \in L^{1}\left(I, \mathbf{R}_{+}\right)$verifying $d\left(D_{C}^{\alpha} x(t), F(t, x(t), y(t))\right) \leq p(t)$ a.e. $(I)$ and $d\left(D_{C}^{\alpha} y(t), G(t, x(t), y(t))\right) \leq q(t)$ a.e. $(I)$. 
Then there exists $(u(),. v()$.$) a solution of problem (1) and (2) satisfying for all t \in I$

$$
|u(t)-x(t)|+|v(t)-y(t)| \leq \frac{\left(M_{1}+M_{3}\right)\|p(.)\|_{1}+\left(M_{2}+M_{4}\right)\|q(.)\|_{1}}{1-\|L(.)\|_{1}} .
$$

Proof. The multifunctions $t \rightarrow F(t, x(t), y(t)), t \rightarrow G(t, x(t), y(t))$ have closed values, are measurable and from Hypothesis 1 one has

$$
\begin{aligned}
& F(t, x(t), y(t)) \cap\left\{D_{C}^{\alpha} x(t)+p(t)[-1,1]\right\} \neq \varnothing \quad \text { a.e. }(I), \\
& G(t, x(t), y(t)) \cap\left\{D_{C}^{\beta} y(t)+q(t)[-1,1]\right\} \neq \varnothing \quad \text { a.e. }(I) .
\end{aligned}
$$

We apply Lemma 3 to find measurable functions $f_{1}(t) \in F(t, x(t), y(t)), g_{1}(t) \in G(t, x(t), y(t))$ a.e. (I) such that

$$
\left|f_{1}(t)-D_{C}^{\alpha} x(t)\right| \leq p(t), \quad\left|g_{1}(t)-D_{C}^{\beta} x(t)\right| \leq q(t) \quad \text { a.e. }(I) .
$$

Define

$$
\begin{aligned}
& u_{1}(t)=\int_{0}^{1} H_{1}(t, s) f_{1}(s) d s+\int_{0}^{1} H_{2}(t, s) g_{1}(s) d s, \quad t \in I \\
& v_{1}(t)=\int_{0}^{1} H_{3}(t, s) f_{1}(s) d s+\int_{0}^{1} H_{4}(t, s) g_{1}(s) d s, \quad t \in I .
\end{aligned}
$$

and one has

$$
\begin{array}{ll}
\left|u_{1}(t)-x(t)\right| \leq M_{1} \int_{0}^{1} p(t) d t+M_{2} \int_{0}^{1} q(t) d t & \forall t \in I, \\
\left|v_{1}(t)-x(t)\right| \leq M_{3} \int_{0}^{1} p(t) d t+M_{4} \int_{0}^{1} q(t) d t & \forall t \in I,
\end{array}
$$

thus, for all $t \in I$

$$
\left|u_{1}(t)-x(t)\right|+\left|v_{1}(t)-x(t)\right| \leq\left(M_{1}+M_{3}\right)|| p(.)\left\|_{1}+\left(M_{2}+M_{4}\right)\right\| q(.) \|_{1}=: K .
$$

We note that it is enough to construct the sequences $u_{n}(),. v_{n}(.) \in C(I, \mathbf{R})$ and $f_{n}(),. g_{n}(.) \in$ $L^{1}(I, \mathbf{R}), n \geq 1$ satisfying

$$
\begin{gathered}
u_{n}(t)=\int_{0}^{1} H_{1}(t, s) f_{n}(s) d s+\int_{0}^{1} H_{2}(t, s) g_{n}(s) d s, \quad t \in I, \\
v_{n}(t)=\int_{0}^{1} H_{3}(t, s) f_{n}(s) d s+\int_{0}^{1} H_{4}(t, s) g_{n}(s) d s, \quad t \in I . \\
f_{n}(t) \in F\left(t, u_{n-1}(t), v_{n-1}(t)\right), \quad g_{n}(t) \in G\left(t, u_{n-1}(t), v_{n-1}(t)\right) \quad \text { a.e. }(I), \\
\left|f_{n+1}(t)-f_{n}(t)\right| \leq l(t)\left(\left|u_{n}(t)-u_{n-1}(t)\right|+\left|v_{n}(t)-v_{n-1}(t)\right|\right) \quad \text { a.e. }(I), \\
\left|g_{n+1}(t)-g_{n}(t)\right| \leq m(t)\left(\left|u_{n}(t)-u_{n-1}(t)\right|+\left|v_{n}(t)-v_{n-1}(t)\right|\right) \quad \text { a.e. }(I) .
\end{gathered}
$$

Assume that this is done; then from (9)-(12) one has

$$
\left|u_{n+1}(t)-u_{n}(t)\right|+\left|v_{n+1}(t)-v_{n}(t)\right| \leq K\left(\|L(.)\|_{1}\right)^{n} \quad \text { a.e. }(I) \quad \forall n \in \mathbf{N} \text {. }
$$

This is true, because if we suppose the last inequality valid for $n-1$ then we have, for almost all $t \in I$,

$$
\begin{aligned}
& \left|u_{n+1}(t)-u_{n}(t)\right| \leq \int_{0}^{1}\left|H_{1}(t, s)\right| \cdot\left|f_{n+1}(s)-f_{n}(s)\right| d s+\int_{0}^{1}\left|H_{2}(t, s)\right| \cdot\left|g_{n+1}(s)-g_{n}(s)\right| d s \leq \\
& M_{1} \int_{0}^{1}\left|f_{n+1}(s)-f_{n}(s)\right| d s+M_{2} \int_{0}^{1}\left|g_{n+1}(s)-g_{n}(s)\right| d s \leq M_{1} \int_{0}^{1} l(t)\left(\left|u_{n}(t)-u_{n-1}(t)\right|+\mid v_{n}(t)-\right. \\
& \left.v_{n-1}(t) \mid\right) d t+M_{2} \int_{0}^{1} m(t)\left(\left|u_{n}(t)-u_{n-1}(t)\right|+\left|v_{n}(t)-v_{n-1}(t)\right|\right) d t \leq K\left(|| L(.)||_{1}\right)^{n-1}\left(M_{1} \int_{0}^{1} l(t) d t\right. \\
& \left.+M_{2} \int_{0}^{1} m(t) d t\right) .
\end{aligned}
$$

Similarly, we get, for almost all $t \in I$,

$$
\left|v_{n+1}(t)-v_{n}(t)\right| \leq K\left(|| L(.) \|_{1}\right)^{n-1}\left(M_{3} \int_{0}^{1} l(t) d t+M_{4} \int_{0}^{1} m(t) d t\right)
$$


and thus, for for almost all $t \in I$ and all $n \in \mathbf{N}$,

$$
\left|u_{n+1}(t)-u_{n}(t)\right|+\left|v_{n+1}(t)-v_{n}(t)\right| \leq K\left(\|L(.)\|_{1}\right)^{n-1} \cdot\|L(.)\|_{1}=K\left(\|L(.)\|_{1}\right)^{n} .
$$

Thus, $\left\{u_{n}().\right\},\left\{v_{n}().\right\}$ are Cauchy in the space $C(I, \mathbf{R})$, therefore, converging uniformly to some $u(.) \in C(I, \mathbf{R})$ and $v(.) \in C(I, \mathbf{R})$. So, by (12), for almost all $t \in I$, the sequences $\left\{f_{n}(t)\right\},\left\{g_{n}(t)\right\}$ are Cauchy in $\mathbf{R}$. Denote by $f(),. g($.$) their the pointwise limit.$

At the same time, one has

$$
\begin{aligned}
& \left|u_{n}(t)-x(t)\right|+\left|v_{n}(t)-y(t)\right| \leq\left|u_{1}(t)-x(t)\right|+\left|v_{1}(t)-y(t)\right|+ \\
& \sum_{i=1}^{n-1}\left(\left|u_{i+1}(t)-u_{i}(t)\right|+\left|v_{i+1}(t)-v_{i}(t)\right|\right) \leq K+\sum_{i=1}^{n} K\left(|| L(.)||_{1}\right)^{i} \leq \frac{K}{1-|| L(.) \mid \|_{1}} .
\end{aligned}
$$

Moreover, from (9), (12) and (13) we obtain for almost all $t \in I$

$$
\begin{aligned}
& \left|f_{n}(t)-D_{C}^{\alpha} x(t)\right|+\left|g_{n}(t)-D_{C}^{\beta} y(t)\right| \leq\left|f_{1}(t)-D_{C}^{\alpha} x(t)\right|+\left|g_{1}(t)-D_{C}^{\beta} y(t)\right|+ \\
& \sum_{i=1}^{n-1}\left(\left|f_{i+1}(t)-f_{i}(t)\right|+\left|g_{i+1}(t)-g_{i}(t)\right|\right) \leq\left|f_{1}(t)-D_{C}^{\alpha} x(t)\right|+\left|g_{1}(t)-D_{C}^{\beta} y(t)\right|+ \\
& \sum_{i=1}^{n-1}(l(t)+m(t))\left(\left|u_{i}(t)-u_{i-1}(t)\right|+\left|v_{i}(t)-v_{i-1}(t)\right|\right) \leq p(t)+q(t)+(l(t)+m(t)) \frac{K}{1-|| L(.) \|_{1}} .
\end{aligned}
$$

In particular, sequences $f_{n}(),. g_{n}($.$) are integrably bounded; thus f(),. g(.) \in L^{1}(I, \mathbf{R})$.

Taking into account Lebesgue's dominated convergence theorem we pass to the limit in (10) and (12) in order to get that $(u(),. v()$.$) is a solution of (1). Similarly, if we pass to the limit in (13) we$ find (8).

In order to finish the proof it remains to realize by induction the construction of sequences $u_{n}(),. v_{n}(),. f_{n}(),. g_{n}($.$) with the properties in (10)-(12).$

We note that the first step is already done. Suppose now that for $N \geq 1, u_{n}(),. v_{n}(.) \in C(I, \mathbf{R})$ and $f_{n}(),. g_{n}(.) \in L^{1}(I, \mathbf{R}), n=1,2, \ldots N$ with (10) and (12) for $n=1,2, \ldots N$ and (11) for $n=1,2, \ldots N-1$ are constructed. The multifunctions $t \rightarrow F\left(t, u_{N}(t), v_{N}(t)\right), t \rightarrow G\left(t, u_{N}(t), v_{N}(t)\right)$ are measurable; as well as the maps $t \rightarrow l(t)\left|u_{N}(t)-u_{N-1}(t)\right|+l(t)\left|v_{N}(t)-v_{N-1}(t)\right|, t \rightarrow m(t)\left|u_{N}(t)-u_{N-1}(t)\right|+$ $m(t)\left|v_{N}(t)-v_{N-1}(t)\right|$ are measurable. Since $F(t, . .$.$) and G(t, . .$.$) are Lipschitz we get$

$$
\begin{aligned}
& F\left(t, u_{N}(t), v_{N}(t)\right) \cap\left\{f_{N}(t)+\left(l(t)\left|u_{N}(t)-u_{N-1}(t)\right|+l(t)\left|v_{N}(t)-v_{N-1}(t)\right|\right)[-1,1]\right\} \neq \varnothing, \\
& G\left(t, u_{N}(t), v_{N}(t)\right) \cap\left\{g_{N}(t)+\left(m(t)\left|u_{N}(t)-u_{N-1}(t)\right|+m(t)\left|v_{N}(t)-v_{N-1}(t)\right|\right)[-1,1]\right\} \neq \varnothing
\end{aligned}
$$

for almost all $t \in I$.

Lemma 3 allows to find measurable selections $f_{N+1}($.$) of F\left(., u_{N}(),. v_{N}().\right)$ and $g_{N+1}($.$) of$ $G\left(., u_{N}(),. v_{N}().\right)$ such that

$$
\begin{aligned}
& \left|f_{N+1}(t)-f_{N}(t)\right| \leq l(t)\left(\left|u_{N}(t)-u_{N-1}(t)\right|+\left|v_{N}(t)-v_{N-1}(t)\right|\right) \quad \text { a.e. }(I) \\
& \left|g_{N+1}(t)-g_{N}(t)\right| \leq m(t)\left(\left|u_{N}(t)-u_{N-1}(t)\right|+\left|v_{N}(t)-v_{N-1}(t)\right|\right) \quad \text { a.e. }(I) .
\end{aligned}
$$

We set $\left(u_{N+1}(),. v_{N+1}().\right)$ as in (10) with $n=N+1$. Thus, $f_{N+1}(),. g_{N+1}($.$) verifies (11) and (12) and$ the proof is finished.

We note that if in Theorem $1 p()=.q()=$.0 it means that $(x(),. y()$.$) is a solution of problem (1)$ and (2) and there is nothing to prove. But the theorem treats the case when mappings $p($.$) and q($. are not zero. This is why $(x(),. y()$.$) is called an almost solution or "quasi" solution. The idea of the$ approach above is to start from an almost solution and to arrive to a solution of the problem.

If we want to obtain an existence result which looks less complicated it is enough to make a particular choice for the almost solution. Namely, we take $x()=.y()=$.0 . We may state the following corollary of Theorem 1. 
Corollary 1. Hypothesis 1 is verified, $d(0, F(t, 0,0)) \leq l(t), d(0, G(t, 0,0)) \leq m(t)$ a.e. $(I), \alpha>\gamma+1$, $\beta>\gamma+1$ and $\|L(.)\|_{1}<1$.

Then there exists $(u(),. v()$.$) a solution of problem (1)-(2) that verifies$

$$
|u(t)|+|v(t)| \leq \frac{\left(M_{1}+M_{3}\right)|| l(.)\|\|_{1}+\left(M_{2}+M_{4}\right)\|m(.)\|_{1}}{1-\|L(.)\|_{1}} \quad t \in I .
$$

Proof. The hypothesis in Theorem 1 is fulfiled, with $x()=.y()=$.0 and $p()=.l(),. q()=.m($.$) .$ It remains to apply Theorem 1 in this case.

A similar result to the one in Corollary 1 may be obtained using a fixed point approach; namely, Covitz and Nadler set-valued contraction principle [10]. But this approach is weaker than Corollary 1 since the values of $F(.,$.$) and G(.,$.$) must be compact and it is not possible to get a priori bounds as$ in (8).

Morever, if in (1), $F$ and $G$ are single-valued maps, Corollary 1 gives an extension of Theorem 3.1 in [8].

Example 1. Consider the following problem

$$
\begin{aligned}
& \begin{cases}D_{C}^{\frac{3}{2}} u(t) \in\left[-\frac{1}{20} \frac{|u(t)|}{1+|u(t)|}, 0\right] \cup\left[0, \frac{1}{20} \frac{|\sin (v(t))|}{1+|\sin (v(t))|}\right] & \text { a.e. }[0,1], \\
D_{C}^{\frac{3}{2}} v(t) \in\left[-\frac{1}{20} \frac{|\cos (u(t))|}{1+|\cos (u(t))|}, 0\right] \cup\left[0, \frac{1}{20} \frac{|v(t)|}{1+|v(t)|}\right] & \text { a.e. }[0,1],\end{cases}
\end{aligned}
$$

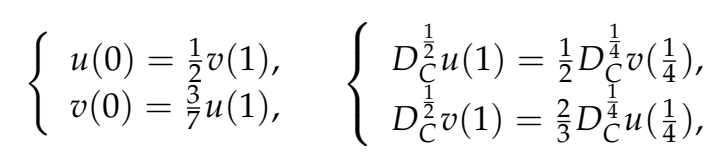

In this case $\alpha=\beta=\frac{3}{2}, \gamma=\xi=\frac{1}{4}, \lambda_{1}=\frac{1}{2}, \lambda_{2}=\frac{1}{3}, \mu_{1}=\frac{3}{7}, \mu_{2}=\frac{2}{3}, F(t, x, y)=\left[-\frac{1}{20} \frac{|x|}{1+|x|}, 0\right] \cup$ $\left[0, \frac{1}{20} \frac{|\sin (y)|}{1+|\sin (y)|}\right]$ and $G(t, x, y)=\left[0, \frac{1}{20} \frac{|y|}{1+|y|}\right] \cup\left[-\frac{1}{20} \frac{|\cos (x)|}{1+|\cos (x)|}, 0\right]$. On one hand,

$$
\begin{gathered}
\sup \{|u| ; u \in F(t, x, y)\} \leq \frac{1}{20}, \quad \sup \{|u| ; u \in G(t, x, y)\} \leq \frac{1}{20} \quad \forall t \in[0,1], x, y \in \mathbf{R}, \\
d_{H}\left(F\left(t, x_{1}, y_{1}\right), F\left(t, x_{2}, y_{2}\right)\right) \leq \frac{1}{20}\left|x_{1}-x_{2}\right|+\frac{1}{20}\left|y_{1}-y_{2}\right| \quad \forall x_{1}, x_{2}, y_{1}, y_{2} \in \mathbf{R}, \\
d_{H}\left(G\left(t, x_{1}, y_{1}\right), G\left(t, x_{2}, y_{2}\right)\right) \leq \frac{1}{20}\left|x_{1}-x_{2}\right|+\frac{1}{20}\left|y_{1}-y_{2}\right| \quad \forall x_{1}, x_{2}, y_{1}, y_{2} \in \mathbf{R}
\end{gathered}
$$

and on the other hand, a simple computation shows that in this example $M_{1} \approx 2.8, M_{2} \approx 2.9, M_{3} \approx 2.2$, $M_{4} \approx 2.5$ and $\left(M_{1}+M_{3}\right) \frac{1}{20}+\left(M_{2}+M_{4}\right) \frac{1}{20} \approx 0.6<1$. Therefore, we are able to apply Corollary 1 in order to deduce the existence of a solution for problem (14) and (15).

In what follows we use the notation: $K(t)=m_{1} l(t)+m_{2} m(t)+m_{3} l(t)+m_{4} m(t), t \in I$.

In the case when we consider integro-multistrip-multipoint boundary conditions, namely problem (3)-(5), we obtain the following existence result.

Theorem 2. Suppose that Hypothesis 1 is verified and $\|K(.)\|_{1}<1$.

Consider $x(.) \in C(I, \mathbf{R}), y(.) \in C(I, \mathbf{R})$ such that $x(0)=x^{\prime}(0)=\ldots=x^{(n-2)}(0)=$ $0, \int_{0}^{1} x(s) d s=\sum_{i=2}^{p_{1}} a_{i-1} \int_{\eta_{i-1}}^{\eta_{i}} y(s) d s+\sum_{j=1}^{q_{1}} b_{j} y\left(\rho_{j}\right), y(0)=y^{\prime}(0)=\ldots=y^{(n-2)}(0)=$ $0, \int_{0}^{1} y(s) d s=\sum_{i=2}^{p_{2}} c_{i-1} \int_{\theta_{i-1}}^{\theta_{i}} x(s) d s+\sum_{j=1}^{q_{2}} d_{j} x\left(\zeta_{j}\right)$ and there exists $r(),. s(.) \in L^{1}\left(I, \mathbf{R}_{+}\right)$with $d\left(D^{\sigma} x(t), F(t, x(t), y(t))\right) \leq r(t)$ a.e. (I) and $d\left(D^{\phi} y(t), G(t, x(t), y(t))\right) \leq q(t)$ a.e. $(I)$. 
Then there exists $(u(),. v()$.$) a solution of problem (3)-(5) which, in addition, satisfies$

$$
|u(t)-x(t)|+|v(t)-y(t)| \leq \frac{\left(m_{1}+m_{3}\right)\|r(.)\|_{1}+\left(m_{2}+m_{4}\right)\|s(.)\|_{1}}{1-\|K(.)\|_{1}} \quad t \in I .
$$

Proof. The proof follows the same pattern as the proof of Theorem 1 and therefore it is omitted.

Corollary 2. Hypothesis 1 is verified, $d(0, F(t, 0,0)) \leq l(t), d(0, G(t, 0,0)) \leq m(t)$ a.e. (I) and $\|K(.)\|_{1}<1$. Then there exists $(u(),. v()$.$) a solution of problem (3)-(5) with the property$

$$
|u(t)|+|v(t)| \leq \frac{\left(m_{1}+m_{3}\right)|| l(.)\left\|_{1}+\left(m_{2}+m_{4}\right)\right\| m(.) \|_{1}}{1-\|K(.)\|_{1}} \quad t \in I .
$$

Proof. It is enough to take in Theorem $2 x()=.y()=$.0 and $r()=.l(),. s()=.m($.$) in order to obtain$ the conclusion of the corollary.

We note that Corollary 2 extends to the set-valued framework Theorem 3.1 in [7].

Example 2. Consider the following problem

$$
\begin{gathered}
\left\{\begin{array}{l}
D^{\frac{5}{2}} u(t) \in\left[-\frac{1}{100} \frac{|u(t)|}{1+|u(t)|}, 0\right] \cup\left[0, \frac{1}{100} \frac{|\sin (v(t))|}{1+|\sin (v(t))|}\right] \text { a.e. }[0,1], \\
D^{\frac{5}{2}} v(t) \in\left[-\frac{1}{100} \frac{|\cos (u(t))|}{1+|\cos (u(t))|}, 0\right] \cup\left[0, \frac{1}{100} \frac{|v(t)|}{1+|v(t)|}\right] \text { a.e. }[0,1],
\end{array}\right. \\
u(0)=u^{\prime}(0)=0, \int_{0}^{1} u(s) d s=\frac{5}{2} \int_{1 / 7}^{1 / 6} v(s) d s+4 \int_{1 / 6}^{1 / 5} v(s) d s+\frac{3}{2} v\left(\frac{1}{4}\right)+2 v\left(\frac{1}{3}\right), \\
v(0)=v^{\prime}(0)=0, \int_{0}^{1} v(s) d s=2 \int_{1 / 3}^{1 / 2} u(s) d s+4 \int_{1 / 2}^{2 / 3} u(s) d s+u\left(\frac{1}{3}\right)+\frac{1}{2} u\left(\frac{2}{3}\right) .
\end{gathered}
$$

We have $\sigma=\phi=\frac{5}{2}, a_{1}=\frac{5}{2}, a_{2}=4, \eta_{1}=\frac{1}{7}, \eta_{2}=\frac{1}{6}, \eta_{3}=\frac{1}{5}, b_{1}=\frac{3}{2}, b_{2}=2, \rho_{1}=\frac{1}{4}$, $\rho_{2}=\frac{1}{3}, c_{1}=2, c_{2}=4, d_{1}=2, d_{2}=\frac{1}{2}, \theta_{1}=\frac{1}{3}, \theta_{2}=\frac{1}{2}, \theta_{3}=\frac{2}{3}, \zeta_{1}=\frac{1}{3}$ and $\zeta_{2}=\frac{2}{3} . F(t, x, y)=$ $\left[-\frac{1}{100} \frac{|x|}{1+|x|}, 0\right] \cup\left[0, \frac{1}{100} \frac{|\sin (y)|}{1+|\sin (y)|}\right], G(t, x, y)=\left[0, \frac{1}{100} \frac{|y|}{1+|y|}\right] \cup\left[-\frac{1}{100} \frac{|\cos (x)|}{1+|\cos (x)|}, 0\right]$ and

$$
\begin{gathered}
\sup \{|u| ; u \in F(t, x, y)\} \leq \frac{1}{100}, \quad \sup \{|u| ; u \in G(t, x, y)\} \leq \frac{1}{100} \quad \forall t \in[0,1], x, y \in \mathbf{R}, \\
d_{H}\left(F\left(t, x_{1}, y_{1}\right), F\left(t, x_{2}, y_{2}\right)\right) \leq \frac{1}{100}\left|x_{1}-x_{2}\right|+\frac{1}{100}\left|y_{1}-y_{2}\right| \quad \forall x_{1}, x_{2}, y_{1}, y_{2} \in \mathbf{R}, \\
d_{H}\left(G\left(t, x_{1}, y_{1}\right), G\left(t, x_{2}, y_{2}\right)\right) \leq \frac{1}{100}\left|x_{1}-x_{2}\right|+\frac{1}{100}\left|y_{1}-y_{2}\right| \quad \forall x_{1}, x_{2}, y_{1}, y_{2} \in \mathbf{R} .
\end{gathered}
$$

By standard calculations, as in the previous example, we get $\left(m_{1}+m_{3}\right) \frac{1}{100}+\left(m_{2}+m_{4}\right) \frac{1}{100}<1$ and applying Corollary 2 we obtain the existence of a solution for problem (16)-(18).

\section{Discussion}

In the present paper we extend existence results obtained for coupled systems of fractional differential equations to the more general problems of coupled systems of fractional differential inclusions. More exactly, if in (1), $F$ and $G$ reduce to functions, then a similar existence property as in Corollary 1 may be found in [8]; namely, Theorem 3.1. Furthermore, if in (3), F and $G$ are single-valued, then Theorem 3.1 in [7] provides a similar result to the one in Corollary 2.

Existence results as in Corollary 1 and Corollary 2 may be obtained, also, by using a fixed point approach, namely; Covitz and Nadler set-valued contraction principle [10]. However, such kind of approach, apart from the requirement that the values of $F(.,$.$) and G(.,$.$) must be compact, does not$ provides a priori bounds as in (8). 
Afterwards, our Theorems 1 and 2 are essential tools in order to obtain qualitative results concerning the solutions of the problems considered: controllability along a given solution and differentiability of trajectories with respect to the initial conditions.

Funding: This research received no external funding

Conflicts of Interest: The author declares no conflict of interest.

\section{References}

1. Băleanu, D.; Diethelm, K.; Scalas, E.; Trujillo, J.J. Fractional Calculus Models and Numerical Methods; World Scientific: Singapore, 2012.

2. Diethelm, K. The Analysis of Fractional Differential Equations; Springer: Berlin, Germany, 2010.

3. Kilbas, A.; Srivastava, H.M.; Trujillo, J.J. Theory and Applications of Fractional Differential Equations; Elsevier: Amsterdam, The Netherlands, 2006.

4. Miller, K.; Ross, B. An Introduction to the Fractional Calculus and Differential Equations; John Wiley: New York, NY, USA, 1993.

5. Podlubny, I. Fractional Differential Equations; Academic Press: San Diego, CA, USA, 1999.

6. Caputo, M. Elasticità e Dissipazione; Zanichelli: Bologna, Italy, 1969.

7. Ahmad, B.; Alsaedi, A.; Ntouyas, S.K. Nonlinear coupled fractional order systems with integro-multistripmultipoint boundary conditions. Int. J. Anal. Appl. 2019, 17, 940-957.

8. Rao, S.N.; Alsemi, M. On a coupled system of fractional differential equations with nonlocal non-separeted boundary conditions. Adv. Differ. Equ. 2019, 2019, 97. [CrossRef]

9. Filippov, A.F. Classical solutions of differential equations with multivalued right hand side. SIAM J. Control 1967, 5, 609-621. [CrossRef]

10. Covitz, H.; Nadler, S.B., Jr. Multivalued contraction mapping in generalized metric spaces. Isr. J. Math. 1970, 8, 5-11. [CrossRef]

11. Cernea, A. Filippov lemma for a class of Hadamard-type fractional differential inclusions. Fract. Calc. Appl. Anal. 2015, 18, 163-171. [CrossRef]

12. Cernea, A. On the existence of solutions for a Hadamard-type fractional integro-differential inclusion. J. Nonlinear Anal. Optim. 2015, 6, 67-72.

13. Cernea, A. On some boundary value problems for a fractional integro-differential inclusion. Nonlinear Funct. Anal. Appl. 2016, 21, 215-223.

14. Cernea, A. On some fractional integro-differential inclusions with nonlocal multi-point boundary conditions. Fract. Differ. Calc. 2019, 9, 139-148. [CrossRef]

15. Aubin, J.P.; Frankowska, H. Set-Valued Analysis; Birkhauser: Basel, Switzerland, 1990. 